\title{
The dynamical model of the planet motions and EPM ephemerides
}

\author{
Elena V. Pitjeva \\ Institute of Applied Astronomy of RAS, Nab Kutuzova 10, RU-191187 St Petersburg, Russia \\ email: evp@ipa.nw.ru
}

Abstract. We discuss the dynamical model of the planet motions and EPM ephemerides.

Keywords. celestial mechanics, astrometry, ephemerides, reference systems, time, solar system

The dynamical model of the motion of planets used for construction of high-precision numerical ephemerides EPM (Ephemerides of Planets and the Moon) at the Institute of Applied Astronomy RAS is presented. The model of EPM2006 ephemerides includes mutual perturbations from the nine planets, the Sun, the Moon, lunar physical libration, perturbations from 301 biggest asteroids, as well as perturbations from the solar oblateness, the massive asteroid ring, and the Kuiper belt objects. The total shift of the barycenter of the solar system due to 19 largest trans-Neptunian objects is $6140 \mathrm{~m}$ within the lifetime of Gaia (2011-2020).

Ephemerides of the planets and the Moon have been produced by numerical integration in the PPN metric over a 170-yr time interval (1880 - 2050) and were oriented onto ICRF by using the ICRF-base VLBI measurements of spacecraft.

The EPM2006 ephemerides have resulted from a least square adjustment to observational data totaling more than 440000 position observations (1913-2005) of different types including radiometric observations of planets and spacecraft, CCD astrometric observations of the outer planets and their satellites, meridian transits and photographic observations. In addition to the TDB-base primary ephemerides, a version of the EPM ephemerides was also constructed in the TCB time scale.

The EPM ephemerides has formed the basis for the Russian 'Astronomical Yearbook' since 2006 and are available at: ftp://quasar.ipa.nw.ru/incoming/EPM2004. 